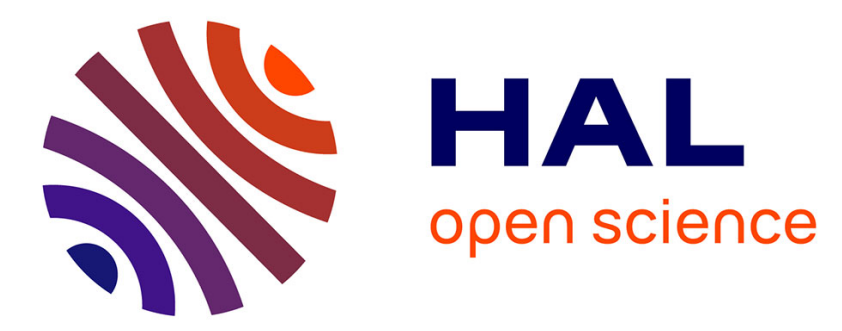

\title{
Reduction strategy for a brake system with local frictional non-linearities - Application for the prediction of unstable vibration modes
}

O Fazio, S Nacivet, Jean-Jacques Sinou

\section{- To cite this version:}

O Fazio, S Nacivet, Jean-Jacques Sinou. Reduction strategy for a brake system with local frictional non-linearities - Application for the prediction of unstable vibration modes. Applied Acoustics, 2015, 91, pp.12 - 24. 10.1016/j.apacoust.2014.11.005 . hal-03257800

\section{HAL Id: hal-03257800 \\ https://hal.science/hal-03257800}

Submitted on 11 Jun 2021

HAL is a multi-disciplinary open access archive for the deposit and dissemination of scientific research documents, whether they are published or not. The documents may come from teaching and research institutions in France or abroad, or from public or private research centers.
L'archive ouverte pluridisciplinaire $\mathbf{H A L}$, est destinée au dépôt et à la diffusion de documents scientifiques de niveau recherche, publiés ou non, émanant des établissements d'enseignement et de recherche français ou étrangers, des laboratoires publics ou privés. 
O. Fazio, S. Nacivet and J-J. Sinou, Reduction strategy for a brake system with local frictional non-linearities - Application for the prediction of unstable vibration modes, Applied Acoustics, 91, 12-24,2015.

doi:10.1016/j.apacoust.2014.11.005

\title{
Reduction strategy for a brake system with local frictional non-linearities - Application for the prediction of unstable vibration modes
}

\author{
O. Fazio ${ }^{1,2, a}$, S. Nacivet ${ }^{2, b}$, and J-.J. Sinou ${ }^{1, c}$ \\ ${ }^{1}$ Laboratoire de Tribologie et Dynamique des Systèmes, UMR CNRS 5513, \\ Ecole Centrale de Lyon, 36 avenue Guy de Collongue 69134 Ecully Cedex, France \\ ${ }^{2}$ PSA Peugeot Citroën, Centre technique de La Garenne Colombes, \\ 18 rue des Fauvelles 92250 La Garenne Colombes, France

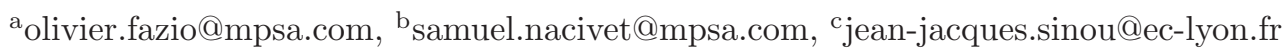

\begin{abstract}
Although the problem of friction-induced vibration has been the subject of many investigations over recent decades, it is still responsible for a large number of nuisances in the field of automotive. This study presents a numerical process based on modal reduction minimizing the size of the model thanks to a specific non-linear modeling at the frictional interface in order to predict the stability analysis of large finite element models that correspond to real automotive braking systems subjected to friction-induced vibrations. In the present study, the effect of the number of contact nodes at the frictional interface will be investigated for various operating conditions. It will be illustrated that an original contact reduction at the frictional interface coupled to Super-Element use can be developed in order to well represent squeal instabilities (i.e the frequencies and the associated unstable modes). It will be demonstrated that the proposed strategy guarantees a high-quality estimation of the stability analysis for the real brake system subjected to friction-induced vibration.
\end{abstract}




\section{Introduction}

The problem of brake squeal in automotive was a subject of great interest for many researchers. The problem of squeal modeling has been extensively undertaken in the past years [1 5] and a lot of numerical and experimental studies including uncertainties [6,7], statistical analysis of brake squeal noise [8], non-linear formulations and behaviors at the frictional interfaces [9 14], chaotic phenomenon [15] or acoustic emissions [16 18 have been formulated and developed in order to explain squeal phenomena and nuisances in the field of automotive. Some researchers have also highlighted the predominant role of the brake pads [19] or the significant impact of the contact surface topography and the frictional contact model 2022$]$ in squeal noise phenomena prediction. Other studies illustrated the fact that introducing uncertainty and robustness concepts during simulations can also improve accuracy of squeal occurrences [7]. However, it is still difficult to predict brake squeal early enough to reduce development costs in a manufacturing process of brake system. So the problem of modeling squeal noise and understanding the mechanisms at the origin of noises are nowadays a current challenge for the scientific community and the automotive industrialists.

In general, there are two main steps toward friction-induced problems: the stability analysis and the non-linear transient analysis. The stability analysis can also be divided into two parts. The first part concerns the non-linear static problem: a sliding equilibrium point is calculated by solving the non-linear static equations for a given brake pressure. Then, the linearized equations of motion are estimated by introducing small perturbations about the sliding equilibrium point into the non-linear system. Finally, the classical Complex Eigenvalue is performed on the linearized system in order to predict the squeal propensity of the brake in a given frequency range. This typical deterministic approach is of common practice and well appreciated in industry. The main advantage of the stability analysis is the computational efficiency in order to estimate the occurrence of self-excited vibrations. However, the main disadvantage and limitation of the stability analysis concerns the underestimation or over-estimation of the unstable modes that can be observed in the non-linear simulation. In fact, the stability analysis can only predict the onset of instability around a given equilibrium point for a non-linear system and so a complete non-linear approach can be more informative [11,23]. Unfortunately, the calculation of the transient and non-linear vibrations are mostly performed by numerical integrations which are rather expensive and requires considerable resources both in terms of computation time and data storage [24,25]. For a large-scale finite element model, numerical integration can become 
computationally so expensive that it is impractical or irrealistic in an industrial context. Based on those facts and according on the size of the problem, industrial studies focus only on the Complex Eigenvalue Analysis (CEA) for linear equivalent brake systems or perform a non-linear approach in order to assess the non-linear self-induced vibrations.

Therefore, some researchers proposed to develop reduced models when finite element models are considered in order to reduce the computational times. For brake system and the squeal prediction, the most widespread reduction method in industry is the Craig-Bampton method [26]. One of the major limitations is associated with the size of interface matrices due to the explicit use of the degrees of freedom at interface. Recently, performances of some others reduced bases built from the component modes or the real coupled modes have been tested by Brizard et al. 27] for the stability analysis of a disc/pads system in sliding contact. They proposed some enriched bases to improve the precision on the calculated complex modes and eigenvalues. Loyer et al. 25] developed also spatial model reduction with different kinds of reduction bases to approximate the non-linear vibrations of a TGV brake system. In these studies, all the physical contact degrees of freedom are kept. Vermot des Roches [24] proposed parametrized reduction called the Component Mode Tuning method (CMT) that use the components free/free modes as explicit degrees of freedom in order to perform non-linear time simulations.

However, few efforts have been dedicated to discuss the possibility to reduce the size of the frictional interface that is one of the main drawback of the classical use of the CraigBampton approach (see for example [12]). So the main contribution of the present study is to propose several improvements in order to reduce the number of contact nodes at the frictional interface. The generation of the reduced non-linear interface model is performed by creating Super-Elements (SE) with reduced node to node contact at the disc/pad interface. The global reduction will then combine the classical Craig-Bampton approach (i.e. a modal reduction strategy) and an implicit reduction at the frictional interface by applying the interface SuperElements (SE) generation. This provides a reduction method that returns accurate results for a drastically reduced finite element system in a lesser time while keeping the non-linear behavior at the frictional interface. The proposed method gives then a great advantage in terms of memory.

The main objective of this study is to propose a strategy based on numerical simulations for predicting the stability analysis of a real brake system subjected to friction-induced vibration. This strategy consists in defining an efficient contact reduction at the frictional interface in order to use a Super-Element for further study of the stability. 
To achieve the previous objectives, the paper is organized as follows: firstly, the brake system under study is presented. Secondly, the proposed reduction method and the global strategy for the generation of the reduced non-linear interface are discussed. More precisely the Super-Element creation and Super-Element assembly are detailed. Finally, application for an industrial representative finite element model is proposed. Results on different cases and

the influence of the number of nodes at the non-linear frictional interface are presented and commented. The efficiency of the reduction strategy is undertaken by performing a stability analysis around a non-linear equilibrium point for various operating conditions.

\section{The brake model under study}

For this study a finite element model of an industrial brake system that has been developed in Abaqus is considered. It can be decomposed in several parts as detailed in Figure 1(a). This system uses a floating caliper technology. The caliper that holds the two brake pads can move with respect to the disc, along a line parallel to the axis of rotation of the disc. During a brake operation, hydraulic pressure is applied on the piston, which pushes the inner brake pad until it makes contact with the disc. Then, reaction force pulls the caliper body with the outer brake pad against the other side of the disc. Considering the finite element model, a volume mesh is realized using 10-node quadratic tetrahedron (see Figure 1(b) . It represents a total of 176956 nodes for 100710 elements.

[Fig. 1 about here.]

Contact and friction modeling are key parameters for squeal simulation. Here, $*$ CONTACT PAIR card is used for node to surface contact modeling. The principal components of the brake system in contact with the pads are the disc, the piston, the caliper and the bracket. For all these contacts, pads are always defined as slave parts.

A linear penalty method is used for the contact constraint enforcement and the classic Coulomb's law is used for all the frictional interfaces. Also depending on the contact considered, separation can be taken into account or not. All these parameters are summed up in Table 1 .

[Table 1 about here.] 


\section{Model reduction method}

Previous research works were carried out to define a model reduction method to apply stability analysis on industrial representative models [24,27]. Alternatively, Villard et al. [28] used an assembly of Super-Elements (SE) with reduced contact interface. However, some questions remained after this study particularly concerning the modeling of the simplified contact interface.

This work was achieved in co-simulation between Abaqus and Matlab. This section will describe the different steps in detail, but it is interesting for the reader comprehension to first sum up the whole process. Firstly, Abaqus simulation is used to define an original reduced contact interface at the disc/pad interface with reduced number of nodes on both sides of pad and disc that will be later used for condensation and node to node contact. Secondly, the static equilibrium is found on this Abaqus model with reduced contact interface. Then, deactivating the disc/pad contact while fixing the displacement of the nodes of this reduced contact interface observed at the static equilibrium allows generating a super-element which is a linearization of this equilibrium configuration. Node to node contact is then used in Matlab at the disc/pad interface when the SE element is used to compute stability analysis with a new cubic contact law for the $\operatorname{disc} / \mathrm{pad}$ interface. In the next paragraphs, all these previous steps are detailed. This section is organized as follows: first the simplified contact modeling will be explained. Then, the super-element creation will be detail to then give some information concerning the Super element assembly and more particularly the method used to define contact force in the node to node contact used for the super-element assembly. Finally some new development brought to improve the reduction method proposed by Villard et al. [28] will be presented.

\subsection{Global strategy and simplified contact modeling}

The idea put forward by Villard et al. [28] is to create an assembly of Super-Elements (SE) at the contact zones defining a node to node contact. Due to the fact that industrial meshing process drives to non-coincident nodes at the frictional interfaces, Villard suggested to generate new contact interfaces for SE assembly using node to node contact with no need to re-mesh the model. Moreover this process allows to reduce the number of degrees of freedom (DOF) at the frictional interfaces of the initial brake system.

The nodes involved in the new contact interface will be later used as reduction nodes for the SE creation. The objective here is to have the smallest number of contact nodes in order 
to reduce the size of the super-element created in retaining this reduced number of contact nodes. Of course reducing the number of nodes kept for computing the contact at the disc/pad interface must not introduce error in the static equilibrium estimation.

This reduced number of nodes involved in the several contacts will be used to later define a node to node contact between the Super-Element (whereas a node to surface contact is used in the reference model). In so far as textcolorredthe reduction of the number of nodes involved in contact must not affect the static solution Villard et al. [28] suggest to distribute the contact forces around these reduced contact nodes. The ${ }^{*}$ DISTRIBUTING and ${ }^{*}$ COUPLING options are therefore used in Abaqus. Figure 2 illustrates this new proposed contact modeling. A reference node is created in the plane of the initial contact surface. It allows to distribute contact force on the nodes of the initial meshed surface involved in the contact. This reference node can not directly be used for the contact computation due to an incompatibility. Due to the fact that SE reduction can not be directly applied to this reference node, an new node must be created for contact force computation and used as a reduction node when SE will be created. An additional spring is created with arbitrary stiffness (i.e. the value of this spring is assumed to be very superior to the local stiffness) to link these two nodes. For the reader comprehension, it can be noticed that these nodes have the same geometric position in the model but they are dissociated on Figure 2 for a better illustration of the reduced contact modeling.

Then, the contact forces (but not the moment) computed at the reduced contact interface are distributed on the coupling nodes of the part surface. Indeed, this reduction method is interesting only if the reduced contact interface computation is relevant and does not generate error on the non-linear static equilibrium. In this strategy it is interesting to exploit the *DISTRIBUTING and *COUPLING cards in Abaqus in order to determine the best set of parameters [29] and decrease the error induced by this reduction and thus improve the previous reduction method.

One of the contribution of this study on simplified contact modeling is to create one SuperElement reducing only the disc/pad interface. All the other interfaces are then linearized during the Super-Element creation. This choice allowed us to reduce the error involved by contact reduction and better understand its effect. Indeed increasing the number of reduced contact interfaces sums up the error involved by reduction. The major drawback is that individual damping can not be considered anymore for each component during Super-Element assembly.

[Fig. 2 about here.] 


\subsection{Super-Element creation and Super-Element assembly}

\subsubsection{Super-Element creation}

The first step concerns the Super-Element creation. Its generation can be summarized as follows: first of all the reduced disc/pad contact interface (i.e a contact with a reduced number of nodes using *DISTIBUTING and *COUPLING cards) is created on the Abaqus model. Then, a nonlinear static analysis is performed on the Abaqus model with reduced contact interface and the displacements at the nodes retained for Super-Element creation are stored. We will see later why it is also interesting to store reduced contact variables (contact force and gap). In a second step, contact at disc/pad interface is deactivated but the displacements measured are still applied to the future reduction nodes with the "*Boundary, FIXED" parameter. This allows to linearize the other contacts of the model, that will not be taken in account in Matlab, at the static equilibrium while generating the Super-Element. Finally a Craig and Bampton reduction [30] is computed and the Super-Element is generated. The reduced number of nodes used in the disc/pad contact interface are kept as reduction nodes.

[Fig. 3 about here.]

\subsubsection{Super-Element assembly}

The second step concerns the Super-Element assembly. It may be noticed that Abaqus in no more used and all the computations are done thanks to Matlab software, as illustrated in Figure 3. This subsection details the method to compute the non-linear static step of the SE assembly at the exact static equilibrium.

The non-linear static system solved by Abaqus is described as follows :

$$
\mathbf{K}_{\mathrm{nl}}\left(\mathbf{U}_{\mathrm{s}}\right) \mathbf{U}_{\mathrm{s}}+\mathbf{F}_{\mathrm{nl}}\left(\mathbf{U}_{\mathrm{s}}\right)=\mathbf{F}_{\mathrm{ext}}
$$

where $\mathbf{U}_{\mathrm{s}}$ corresponds to the static equilibrium position. $\mathbf{F}_{\text {ext }}$ represents the external force due to the external pressure applied to the piston and the caliper representing a brake operation. $\mathbf{K}_{\mathrm{nl}}$ is the stiffness matrix due to the structural components and the three contact interfaces Piston/Pad, Bracket/Pad and Caliper/Pad. $\mathbf{F}_{\mathrm{nl}}$ contains the non-linear forces at the frictional interface between the disc and the pad.

As the Super-Element is created in a perturbation step, the system linearized around the static equilibrium can be written as follows:

$$
\mathbf{K}_{\mathrm{nl}, \mathbf{U}_{\mathrm{s}}} \mathbf{U}_{\mathrm{s}}+\mathbf{F}_{\mathrm{nl}}\left(\mathbf{U}_{\mathrm{s}}\right)=\mathbf{F}_{\text {ext }}
$$


where $\mathbf{K}_{\mathrm{nl}, \mathbf{U}_{\mathrm{s}}}$ defines the linearized stiffness matrix at the vicinity of the static equilibrium $\mathbf{U}_{\mathrm{s}}$. All the contacts, except for the disc/pad interface, are linearized as a constant value in the stiffness matrix.

The local stability is studied introducing a perturbation $\Delta \mathbf{U}$ around the static equilibrium:

$$
\mathbf{K}_{\mathrm{nl}, \mathbf{U}_{\mathrm{s}}}\left(\mathbf{U}_{\mathrm{s}}+\Delta \mathbf{U}\right)+\mathbf{F}_{\mathrm{nl}}\left(\mathbf{U}_{\mathrm{s}}+\Delta \mathbf{U}\right)=\mathbf{F}_{\mathrm{ext}}
$$

Considering the Matlab implementation, the linearized system at the static non-linear equilibrium point is described in the following equation:

$$
\mathbf{K}_{\mathrm{nl}, \mathbf{U}_{\mathrm{s}}} \mathbf{U}_{\mathrm{s}}+\mathbf{F}_{\mathrm{nl}, \text { matlab }}\left(\mathbf{U}_{\mathrm{s}}\right)=\mathbf{F}_{\mathrm{ext}}
$$

where $\mathbf{F}_{\text {nl,matlab }}$ corresponds to the non-linear contact as the disc/pad interface that will be described in the next section. $\mathbf{K}_{\mathrm{nl}, \mathbf{U}_{\mathrm{s}}}$ is obtained thanks to the Super-Element creation in Abaqus. The only difference with equation 2 is the way to compute the non-linear force involved in disc/pad contact. According to the strategy defined above this contact is now a node to node contact between condensation nodes. As the calculated linearized equilibrium points for Abaqus software and Matlab software are the same, an equivalence of the contact force at the disc/pad interface for the non-linear equilibrium point has to be performed:

$$
\mathbf{F}_{\text {matlab }}\left(\mathbf{U}_{\mathrm{s}}\right)=\mathbf{F}_{\mathrm{nl}}\left(\mathbf{U}_{\mathrm{s}}\right)
$$

The next paragraph will develop the solution proposed here to respect the relation detailed in the previous equation.

\subsubsection{Generation of the contact force}

Considering experimental data, Coudeyras [12] chose a cubic stiffness for the disc/pad non-linear contact. For each contact node, its formulation is given in Eq[6] and 7

$$
\begin{gathered}
\mathbf{F}_{\text {matlab }}= \begin{cases}k_{l} \delta+k_{n l} \delta^{3} & \text { if } \delta>0 \\
\mathbf{0} & \text { otherwise }\end{cases} \\
\delta=\mathbf{U}_{\mathbf{i}}-\mathbf{U}_{\mathbf{j}}
\end{gathered}
$$

where $k_{l}$ and $k_{n l}$ are respectively the linear and the non-linear stiffnesses and $\mathbf{U}_{\mathrm{i}}$ and $\mathbf{U}_{\mathrm{j}}$ the displacements of the coincident nodes $\mathrm{i}$ and $\mathrm{j}$ from the master and slave sides of the contact respectively. The subscript matlab indicates that the non-linear forces computation is performed on the Matlab environment. 
The main advantage of this choice is that this formulation is easy to linearize for further stability analysis. As said earlier we need to ensure the continuity in the contact force estimation between Abaqus and Matlab software. With two different contact formulations the only way to respect this constraint is to find an equivalent contact penetration for each node to node contact defined in Matlab in order to match the normal contact force value obtained with Abaqus software. Knowing the value of Abaqus normal contact force for each reduced node and the cubic stiffness parameters $k_{l}$ and $k_{n l}$, it is easy to solve the following relation

$$
\mathbf{F}_{\text {matlab }}(\delta+\xi)=\mathbf{F}_{\mathrm{nl}}(\delta)
$$

where $\mathbf{F}_{\mathrm{nl}}$ are the contact forces computed earlier with Abaqus software and $\delta$ the contact penetration associated to the static equilibrium point that has been previously estimated using Abaqus software. As said earlier this contact is computed using a linear penalty method in Abaqus software. Then the corrective term $\xi$ in node to node contact penetration can be easily found.

Figure 3 details all the steps explained earlier of our model reduction strategy.

\subsection{Interface reduction study: Radius of influence, Weighting methods and Triangular distribution}

In this section the simplified contact modeling defined in 3.1 will be detailed. By default,using *DISTRIBUTING and *COUPLING cards to couple a single node to a meshed surface transfers the forces computed at the reference node to all the nodes of the meshed surface. An interesting option is to limit the distribution in a spherical region centered on the reference node by defining a radius of influence. In this case the forces computed at the reference node are distributed only to the nodes of the surface that fall inside this spherical region. Another interesting parameter is the control over transmission through weight factors specified at each coupling nodes which allow the forces transferred to the coupling nodes to vary inversely with the radial distance from the reference node. For the proposed contact reduction, the radius of influence and weighting method are the main parameters to be fitted in order to have a smooth variation of contact force over the coupling nodes.

In Abaqus 6.10 the influence of the mesh size is also taken into account in the weighting factor computation but it was not considered in this study. To facilitate the understanding of the present study, the classic polynomials defined below as weighting method will be used

$$
w_{i}=1-\frac{r_{i}}{R}
$$




$$
\begin{gathered}
w_{i}=1-\left(\frac{r_{i}}{R}\right)^{2} \\
w_{i}=1-3 \cdot\left(\frac{r_{i}}{R}\right)^{2}+2 \cdot\left(\frac{r_{i}}{R}\right)^{3}
\end{gathered}
$$

where $r_{i}$ is the distance between the reference node and the $i^{\text {th }}$ coupling node, $R$ the radius of influence and $w_{i}$ the weight factor computed.

As said before, reducing the number of contact nodes and distributing force must not affect the contact force mapping on the interface. Indeed it is not recommended to concentrate the forces at the vicinity of the new reduced contact nodes and thus to modify the static equilibrium. Considering these three weighting methods it is interesting to find for each of them the most interesting radius of influence in order to ensure an uniform contact force distribution that matches the most with the initial non-reduced contact. As distribution is made in a circular region centered on the reference node, it is interesting to define the reduced contact nodes group as an isometric grid to reduce voids in distribution (see Figure 44). Thus optimal radius will depend on the triangle size of the isometric grid. So the optimum was calculated for an academic example of a simple triangle of three nodes representing the elementary pattern of our isometric grid. Weighting coefficient mapping inside this triangle of reduced nodes is computed in Matlab for a triangle length of $1 \mathrm{~mm}$. As an equal force on each node of the triangle is applied, the optimal radius that offers an homogeneous force distribution has to be found for each weighting method, which means a computed weight factor centered on 1 inside this triangle. Results are given on Figure 5 and Table 2 ,

It seems interesting to use a cubic distribution that offers the smallest standard deviation. Whatever the number of reduced contact nodes and so the size of the triangle chosen later in our reduction it will be easy to find, proportionally to these previous results, the radius of distribution that needs to be used. However the edge of contact surface might be subjected to boundary effect of the distribution.

[Fig. 4 about here.]

[Fig. 5 about here.]

[Table 2 about here.]

\section{Application for the reduced industrial model}

This section presents the application of the reduction strategy defined above to the industrial brake model. The main objective of this section is to illustrate the efficiency of the proposed 
methodology to build a reduced finite element model while keeping a good prediction on the three main instabilities observed on the reference Abaqus model.

\subsection{Contact formulation and reduced models characteristics}

First of all, it must be noted that the proposed strategy and reduction formulation is not compatible with the ${ }^{*}$ CONTACT PAIR card used for contact in Abaqus due to the fact that the methodology involves node-based surface creation which can not be defined as the master surface of a contact pair in Abaqus. One solution could be to directly define node to node contact between reduced node of both side directly in Abaqus with *GAP elements with friction. However, this strategy does not allow us to generate unsymmetrical terms in matrices which is the key point for stability analysis. So we decided to create reduced contact nodes only for the pad: coincident nodes on the disc volume mesh are imposed and a node to surface formulation with *CONTACT PAIR card is kept. The reduced contact nodes and their coincident nodes on disc mesh will then be used for condensation and node to node contact will be defined later in Matlab between them.

To illustrate the effectiveness of the proposed strategy and discuss the impact of the size of the proposed reduction, several Abaqus models with several triangle size for disc/pad contact interface reduction will be investigated. Table 3 sums up the characteristics of the four models under consideration. Figure 6 gives an insight of the reduced contact nodes layout over the pad surface. As point of comparison, the industrial Abaqus model contains 1370 nodes on the surface of each pad.

In the following of the study, the four Abaqus models with reduced contact interface will be called Abaqus12, Abaqus44, Abaqus104 and Abaqus212 in reference to the number of reduced contact elements they contain.

[Table 3 about here.]

[Fig. 6 about here.]

\subsection{Abaqus reduced models: non-linear static analysis}

As previously explained in [31, the complex eigenvalue analysis is based on the determination of eigenvalues for the linearized system around the non-linear static equilibrium. The first step to evaluate the performance of the reduced contact interface is to compare some static quantities between the Abaqus reference and the four reduced models (Abaqus12, Abaqus44, 
Abaqus104 and Abaqus212): the estimation of the contact state at the frictional interface and the constraints distribution will be undertaken.

\subsubsection{Contact state}

The first proposed comparison between the four reduced models and the reference model corresponds to the contact state when establishing the non-linear static equilibrium. So, a non-linear static analysis for a friction coefficient value of $\mu=0.5$ (at the disc/pad interface) is computed. Figures $7(\mathrm{a}-\mathrm{h})$ show the results for the four reduced models (i.e. Abaqus12, Abaqus44, Abaqus104 and Abaqus212, respectively). Contact state at each reduced node is represented by a circle (respectively by a plus sign) if the contact is open (respectively closed). Moreover, the contact map of the reference model is superimposed for each reduced models: the green and blue colors represent the closed and opened contact

As illustrated in Figures 7(a-b), the first reduced model Abaqus12 (with only 6 nodes by pad) is not sufficient to respect an appropriate contact state at the static equilibrium point: all the reduced contact nodes are closed, whereas the reference model indicates both opened and closed contact area. Refining the number of reduced contact nodes allows to represent the variation in the contact state for the reference model. For example, it clearly appears that the reduced model Abaqus212 is in perfect agreement with the reference model, as indicated in Figures $7(\mathrm{~g}-\mathrm{h})$.

Despite one or two nodes at the boundary between opened and closed contact zone the numerical results with the other two reduced models (i.e Abaqus44 and Abqus104) are consistent with those of the reference model (see Figures $7(\mathrm{c}-\mathrm{d})$ and Figures $7(\mathrm{e}-\mathrm{f})$, respectively). This illustrates the fact that the number of nodes at the frictional contact interface can be drastically reduced in order to estimate the contact states by applying an appropriate strategy.

[Fig. 7 about here.]

\subsubsection{Constraints}

The second verification to illustrate the efficiency of the proposed reduction during consideration of the non-linear static analysis is to investigate the evolution of the constraint field at the frictional interface.

Firstly, it may be noted that the general node to surface contact is defined such as each slave node interacts with a projection point on the master surface. If the projection of the slave 
nodes on the master surface falls inside an element facet, the transmitted load is shared by the nodes of this facet. With the coincident mesh the projected node falls at the exact position of a node of the master surface. In this last case, the transmitted load is shared by the coincident master node and all of the master surface nodes that share an adjacent surface facet with that node, as illustrated in Figure 8, In one way we can say that the coincident node smooths the contact force distribution on the master surface.

In order to be able to compare the constraint fields at the contact interface (i.e. Von Mises stress), the contact force calculated at the reduced contact node of the pad side is then distributed through the coupling element. Then, the constraint fields at the contact interface is estimated. First of all, non-linear static analysis for a friction coefficient $\mu=0.5$ is computed. Results of the constraint fields are presented for both the reference model and the reduced model Abaqus212 on Figures 9(e-h) and Figures 9(i-l), respectively. The color scales given in Figures 9 (a-d) are based on the reference model and according to the finite element model units, constraint value is given in MPa. It can be observed that the constraint fields for the reference model and the reduced model Abaqus212 are very similar. Even if the disc mesh on the reduced model differs from the reference models due to the imposed coincident nodes a good global coincidence in the constraint field can be noticed for each interface of the brake system. Then, numerical results at the inner pad interface for two different friction coefficients $\mu=0.2$ and $\mu=0.8$ are also indicated in Figures 10. Whatever the friction coefficient and the evolution of the associated non-linear static equilibrium, the constraint fields that are calculated via the reduced model Abaqus212 are consistent with those of the reference. Results at the outer pad, outer and inner disc surfaces (not presented in this study for $\mu=0.2$ and $\mu=0.8$ ) corroborate these conclusions.

Finally, it should be interesting to observe the influence of the number of reduced contact nodes on the constraint fields. Figures 11 give the constraint fields for the outer surface of the disc with the four reduced models (i.e. Abaqus12, Abaqus44, Abaqus104 and Abaqus212) with a friction coefficient $\mu=0.5$.

If the number of reduced contact nodes is very small (i.e. 6 and 22 nodes per pad for Abaqus12 and Abaqus44), difference with the reference model is noticeable (see Figures 11(b-c) for Abqus12 and Abaqus44 in comparison with Figure11(a) for the reference). Constraint peaks are concentrated at the coincident nodes on the disc surface. For the reduced models Abaqus12 and Abaqus44, the distance between two reduced contact nodes is larger than the mesh size of the disc, thus contact force are only computed on a really local zone around each slave node 
projection. Then, increasing the number of reduced contact nodes (i.e the reduced models Abaqus104 or Abaqus212) allows a better distribution of the constraint field. So, the constraint field estimated by using these two reduced models are in perfect agreement with the reference model, as illustrated in Figures 11(d) (for Abaqus104) and 9(k). For the reader comprehension, results for the reference model are given in Figure 9(g). Moreover, the other numerical results (not presented in this study) at the inner/outer pads and the inner/outer disc surfaces for the four reduced models (i.e. Abaqus12, Abaqus44, Abaqus104 and Abaqus212) and for the three friction coefficients (i.e. $\mu=0.2, \mu=0.5$ and $\mu=0.8$ ) lead to the same conclusion.

[Fig. 8 about here.]

[Fig. 9 about here.]

[Fig. 10 about here.]

[Fig. 11 about here.]

\subsection{Abaqus reduced models: stability analysis}

In the previous section, it was illustrated that it is possible to drastically reduced the number of contact nodes at the frictional interface while being able to estimate the contact states and the constraint field at the static non-linear equilibrium.

The results of stability analysis between the reference Abaqus model and the four reduced models Abaqus12, Abaqus44, Abaqus104 and Abaqus212 are discussed. A classical complex eigenvalue analysis, with a friction coefficient varying from $\mu=0.1$ to $\mu=0.9$ with a step size of 0.1 , is performed and we focus on the instabilities in the $0-5 \mathrm{kHz}$ range. a global overview of the stability analysis is given in Figure 12, Moreover, evolution of the frequencies and real parts of the three unstable modes are drawn for the reference model and the four reduced models on Figures 13.

It clearly appears that the reduced model Abaqus12 is not sufficient to predict instabilities beyond $4 \mathrm{kHz}$. Indeed, we observe that the unstable mode at $3.8 \mathrm{kHz}$ disappears for $\mu=0.4$ (as indicated in Figure 13(e)) which suggests that a change in contact status can completely modify the characteristics of the brake system. This result is not surprising because of the inconclusive results of this reduced model to correctly estimate the static states, as previously seen in the previous section. Concerning the second reduced model Abaqus44, the three instabilities of interest (at $1900 \mathrm{~Hz}, 3800 \mathrm{~Hz}$ and $4900 \mathrm{~Hz}$ ) are predicted, even if a little delay for the first and 
second unstable modes (at $1.9 \mathrm{hz}$ and $3.9 \mathrm{kHz}$ ) can be observed. Moreover, limitation of this reduced model Abaqus44 can be highlighted by seeing evolution of the third unstable mode for high friction coefficients: indeed a decrease in frequency and a margin in real part evaluation are clearly observed in Figures 13 (c) and (f), respectively.

Finally, the two last reduced model (i.e. Abaqus104 and Abaqus212) correctly approximate the three unstable modes on the frequency range of interest for both the value of real part and the value of the associated frequency, as illustrated in Figure 12 and Figures 13, Increasing the number of reduction nodes between Abaqus104 and Abaqus212 does not lead to significant improvement. In order to have a more detailed comparison of these two reduced models Abaqus104 and Abaqus212, the mean error on the three instabilities of interest is estimated for all the values of the friction coefficient in the range of interest (i.e. from $\mu=0.1$ to $\mu=0.9$ with a step size of 0.1 ). The mean error is defined by

$$
\varepsilon_{\text {tot }}=\sum_{j=1}^{9} \sum_{i=1}^{2} \frac{1}{M} \frac{\left|f_{\mu_{j}, i}^{r e d}-f_{\mu_{j}, i}^{r e f}\right|}{f_{\mu_{j}, i}^{r e f}}
$$

where $\mu_{j}$ corresponds to the value of the friction coefficient given by $\mu_{j}=0.1 * j$. Considering one instability, $f_{\mu_{j}, i}^{r e f}$ (respectively $f_{\mu_{j}, i}^{r e d}$ ) is the frequency of the $i^{\text {th }}$ of the two modes that couple at the friction value $\mu$ for the Abaqus reference model (respectively reduced model). $M$ stands for the number of friction coefficient for which a complex eigenvalue analysis is computed for the stability analysis. The minimum and maximum errors are also defined by

$$
\begin{aligned}
\varepsilon_{\min } & =\mu_{j}^{\min }\left(\sum_{i=1}^{2} \frac{\left|f_{\mu_{j}, i}^{r e d}-f_{\mu_{j}, i}^{r e f}\right|}{f_{\mu_{j}, i}^{r e f}}\right) \\
\varepsilon_{\max } & ={ }_{\mu}^{\max }\left(\sum_{i=1}^{2} \frac{\left|f_{\mu_{j}, i}^{r e d}-f_{\mu_{j}, i}^{r e f}\right|}{f_{\mu_{j}, i}^{r e f}}\right)
\end{aligned}
$$

All these results are summed up on Table 4 and Table 5 . Whatever the instability considered the error does not exceed two percent, the performance of the contact reduction is clearly highlighted here. Considering the mean error, the convergence of the reduction strategy is observed and the remaining error can be explained by the boundary effect of the distribution. In conclusion, results on stability analysis show that the reduced model Abaqus104 fits perfectly on the whole frequency range of interest (i.e. between $0-6 \mathrm{kHz}$ ) in order to predict all the instabilities (in terms of frequency and real part of unstables modes) while allowing to reduce significantly the number of contact nodes at the disc/pad interface. 
[Fig. 12 about here.]

[Fig. 13 about here.]

[Table 4 about here.]

[Table 5 about here.]

\subsection{Super-Element assembly: stability analysis}

The previous results confirmed that reducing the disc/pad contact interface with 104 contact nodes is sufficient to predict all the instabilities on the $0-5 \mathrm{kHz}$ range of interest. I $\mathrm{t}$ is worth remembering that the number of reduction nodes at the frictional interface can vary with the system under study due to the mode shape of unstable modes and the frequency range of interest.

Now we propose to illustrate the efficiency of the second part of the global strategy based on the Super-Element assembly. As a reminder, all these developments have been previously explained in Section 3 .

First of all, based on the retained reduced model Abaqus104, a Super-Element (SE) for a friction coefficient of $\mu=0.5$ at the disc/pad interface is generated. Real modes over $0-12 \mathrm{kHz}$ are kept for the Super-Element creation so that its validity can be assumed over $0-6 \mathrm{kHz}$, our frequency range of interest. It represents a total of 135 modes. Once the SE created, assembled in Matlab with contact force matched and linearized around the non-linear static equilibrium, a complex eigenvalue analysis is undertaken. The results between the Super-Element assembly (SE assembly) and the reduced model Abaqus104 are compared in Figure 14, It can be noticed that the stability analysis is performed on the whole friction range of interest (i.e. from $\mu=0.1$ to $\mu=0.9$ ) with only a Super-Element created at $\mu=0.5$. It is observed that the global reduction based on the Super-Element assembly is not able to reproduce correctly the prediction of unstable vibration modes (both in terms of frequency and real parts). For example it can be seen that for high friction coefficient, the third instability (around $4.7 \mathrm{kHz}$ ) disappears with the reduced model via SE assembly. These results shows that a Super-Element remains valid only at the vicinity of the non-linear static equilibrium point. More particularly, varying the friction coefficient at the disc/pad interface changes the static equilibrium on the Abaqus reference model as indicated in Figures 15. Such changes in the non-linear static equilibrium are not taken in account if the same Super-Element created at $\mu=0.5$ is used for the whole stability 
analysis. This can explain the differences observed between the reduced model with SE assembly and the reduced model Abaqus104.

Considering these first previous results based on the Super-Element assembly, we propose to generate a Super-Element for each step of the friction coefficient (i.e. from $\mu=0.1$ to $\mu=0.9$ with a step size of 0.1 ). Then a complex eigenvalue analysis is performed for each friction coefficient by using the associated Super-Element assembly. As illustrated in Figures 16, results for SE assembly of both frequencies and real parts of the three unstable modes are in agreement fit with those of the reduced model Abaqus104. Good enough with the evolution of the results on the corresponding Abaqus104 model, even if a sudden rise in frequency of the third unstable mode starting at $\mu=0.6$ can be noticed.

Finally, the mean error (given in equation 12), the minimum and maximum errors (given in equations 13 and 14, respectively) are calculated for the SE assembly. The two strategies defined for SE assembly (by using only one Super-Element for the whole stability analysis or by estimating one Super-Element for each friction coefficient) are compared to the reduced model Abaqus104. We recall that this reduced model Abaqus104 was used for the SE generation. Results are given in Tables 6 and 7. It clearly shows the interest of creating one SE for each friction coefficient value. With this strategy the error never exceeds five percent whatever the unstable mode of interest. In conclusion, these results illustrate the efficiency of the global strategy based on both nodal interface reduction and Super-Element generation.

[Fig. 14 about here.]

[Fig. 15 about here.]

[Fig. 16 about here.]

[Table 6 about here.]

[Table 7 about here.]

\section{Conclusion}

The global reduction strategy that is based on a nodal reduction, Super-Elements generation and a Craig and Bampton modal reduction at the frictional interface, is proposed for an industrial non-linear brake system model. One of the advantage of the proposed strategy is based on an efficient contact reduction method for the disc/pad interface that allows a Craig \& Bampton 
reduction to be used for further stability analysis. Final number of variables on this reduced model is about 1500 .

This strategy allows to predict correctly both the non-linear static equilibrium (i.e. the contact states and the constraint field) and the stability analysis at this equilibrium. It is shown that the Super-Element generation needs to be performed for each set of input parameters because of the possible influence of the static equilibrium position on the stability analysis.

Based on these results, future work on the calculation of self-excited vibrations can be investigated by using reduced models via Super-Elements generation.

\section{Acknowledgment}

This work was achieved within Stellab program. The authors would like to thank the PSA Peugeot Citroën staff for their assistance.

\section{References}

[1] N.M. Kinkaid, O.M. O'Reilly, and P. Papadopoulos. Automotive disc brake squeal. Journal of Sound and Vibration, 267:105-166, 2003.

[2] R.A. Ibrahim. Friction-induced vibration, chatter, squeal, and chaos part 1: mechanics of contact and friction. Am Soc Mech Eng Appl Mech Rev, 47(7):209-226, 1994.

[3] R.A. Ibrahim. Friction-induced vibration, chatter, squeal, and chaos part 2: dynamics and modeling. Am Soc Mech Eng Appl Mech Rev, 47(7):227-263, 1994.

[4] H. Ouyang, J.E. Mottershead, D.J. Brookfield, S. James, and M.P. Cartmell. Methodology for the determination of dynamic instabilities in a car disc brake. International Journal of Vehicle Design, 23(3):241-262, 2000.

[5] H. Ouyang, W. Nack, Y. Yuan, and F. Cocheteux. Numerical analysis of automotive disc brake squeal: a review. International Journal of Vehicle Noise and Vibration, 1(3):207-231, 2005 .

[6] E. Sarrouy, O. Dessombz, and J.-J. Sinou. Piecewise polynomial chaos expansion with an application to brake squeal of linear brake system. Journal of Sound and Vibration, 332:577-594, 2013. 
[7] A. Heussaff, L. Dubar, T. Tison, M. Watremez, and R. F. Nunes. A methodology for the modelling of the variability of brake lining surfaces. Wear, 289:145-159, 2012.

[8] S. Oberst and J.C.S. Lai. Statistical analysis of brake squeal noise. Journal of Sound and Vibration, 330(12):2978-2994, 2011.

[9] F. Massi, L. Baillet, O. Giannini, and A. Sestieri. Brake squeal: linear and nonlinear numerical approaches. Mechanical Systems and Signal Processing, 21(6):2374-2393, 2007.

[10] A. Akay, O. Giannini, F. Massi, and A. Sestieri. Disc brake squeal characterization through simplified test rigs. Mechanical Systems and Signal Processing, 23(8):2590-2607, 2009.

[11] J.-J. Sinou. Transient non-linear dynamic analysis of automotive disc brake squeal - on the need to consider both stability and non-linear analysis. Mechanics Research Communications, 37(1):96-105, 2010.

[12] N. Coudeyras, S. Nacivet, and J.-J. Sinou. Periodic and quasi-periodic solutions for multiinstabilities involved in brake squeal. Journal of Sound and Vibration, 328(4):520-540, 2009 .

[13] J.-J. Sinou, N. Coudeyras, and S. Nacivet. Study of the non-linear stationary dynamic of single and multi-instabilities for disk brake squeal. International Journal of Vehicle Design, 51(1):207-222, 2009.

[14] H. Festjens, G. Chevallier, F. Renaud, J.-L. Dion, and R. Lemaire. Effectiveness of multilayer viscoelastic insulators to prevent occurrences of brake squeal: A numerical study. Applied Acoustics, 73(11):1121-1128, November 2012.

[15] S. Oberst and J.C.S. Lai. Chaos in brake squeal noise. Journal of Sound and Vibration, 330(5):955-975, 2011.

[16] S. Oberst, J. C. S. Lai, and S. Marburg. Guidelines for numerical vibration and acoustic analysis of disc brake squeal using simple models of brake systems. Journal of Sound and Vibration, 332(9):2284-2299, 2013.

[17] K. Soobbarayen, S. Besset, and J.-J. Sinou. Noise and vibration for a self-excited mechanical system with friction. Applied Acoustics, 74(10):1191-1204, 2013. 
[18] K. Soobbarayen, S. Besset, and J-J. Sinou. A simplified approach for the calculation of acoustic emission in the case of friction-induced noise and vibration. Mechanical Systems and Signal Processing, 50:732-756, 2015.

[19] F. Renaud, G. Chevallier, J-L. Dion, and G. Taudiere. Motion capture of a pad measured with accelerometers during squeal noise in a real brake system. Mechanical Systems and Signal Processing, 33:155-166, 2012.

[20] A.R. AbuBakar and H. Ouyang. Complex eigenvalue analysis and dynamic transient analysis in predicting disc brake squeal. International Journal of Vehicle Noise and Vibration, 2:143-155, 2006.

[21] A.R. AbuBakar and H. Ouyang. A prediction methodology of disk brake squeal using complex eigenvalue analysis. International Journal of Vehicle Design, 46(4):416-435, 2008.

[22] F. Massi, Y. Berthier, and L. Baillet. Contact surface topography and system dynamics of brake squeal. Wear, 265(11-12):1784-1792, 2008.

[23] J.-J. Sinou, A. Loyer, O. Chiello, G. Mogenier, X. Lorang, F. Cocheteux, and S. Bellaj. A global strategy based on experiments and simulations for squeal prediction on industrial railway brakes. Journal of Sound and Vibration, 332(20):5068-5085, 2013.

[24] G. Vermot des Roches. Frequency and time simulation of squeal instabilities - Application to the design of industrial automotive brakes. PhD thesis, Ecole Centrale Paris, 2011.

[25] A. Loyer, J.-J. Sinou, O. Chiello, and X. Lorang. Study of nonlinear behaviors and modal reductions for friction destabilized systems. application to an elastic layer. Journal of Sound and Vibration, 331(5):1011-1041, 2012.

[26] R. J. Craig. A review of time-domain and frequency domain component mode synthesis methods. International Journal of Analytical and Experimental Modal Analysis, 2(2):59-72, 1987.

[27] D. Brizard, O. Chiello, and J.-J. Sinou X. Lorang. Performances of some reduced bases for the stability analysis of a disc/pads system in sliding contact. Journal of Sound and Vibration, 330(4):703-720, 2011. 
[28] P. Villard, S. Nacivet, and J.-J. Sinou. Superelement reduction of industrial finite element brake system for a constrained harmonic balance method. In INTER-NOISE and NOISECON Congress and Conference Proceedings, pages 3142-3151, 2012.

[29] Abaqus Analysis User's Manual, chapter 31 Constraints.

[30] N. Coudeyras, J.-J. Sinou, and S. Nacivet. A new treatment for predicting the self-excited vibrations of nonlinear systems with frictional interfaces: The constrained harmonic balance method, with application to disc brake squeal. Journal of Sound and Vibration, 319(3):1175-1199, 2009.

[31] J-J. Sinou, F. Thouverez, and L. Jezequel. Methods to reduce non-linear mechanical systems for instability computation. Archives of Computational Methods in Engineering: State of the Art Reviews, 11(3):257-344, 2004. 


\section{List of Figures}

$1 \quad$ Brake svstem details . . . . . . . . . . . . . . . . . . . . . 23

$2 \quad$ The reduced contact mode . . . . . . . . . . . . . . . . . . . . . . . . . . 24

$3 \quad$ Global strategv . . . . . . . . . . . . . . . . . . . . . . . . 25

$4 \quad$ Reduced contact nodes lavout . . . . . . . . . . . . . . . . . . . 26

$5 \quad$ Weight factor distribution . . . . . . . . . . . . . . . . . 27

6 Reduced contact nodes lavout over the pad surface for several reduced model . . 28

7 Contact state comparison for each reduced model . . . . . . . . . . . . . . . . . 29

$8 \quad$ Contact force distribution . . . . . . . . . . . . . . . . . . 30

$9 \quad$ Constraint field comparison between Abaqus models $\mu=0.5$. . . . . . . . . . . 31

10 Constraint field comparison at the inner $\mathrm{pad} \ldots \ldots \ldots \ldots \ldots . \ldots . \ldots 32$

$11 \quad$ Vom Mises stress comparison on outer disc surface . . . . . . . . . . . . . . 33

12 Global evolution of the three main instabilities versus friction . . . . . . . . . 34

13 Evolution of the frequencies and the real parts against friction coefficient $\ldots 35$

14 Evolution of the frequencies and the real parts. unique Super-Element . . . . . 36

15 Outer $\mathrm{pad} /$ Caliper contact status evolution versus friction . . . . . . . . . 37

16 Evolution of the frequencies and the real part. multiple Super-Elements . . . . 38 


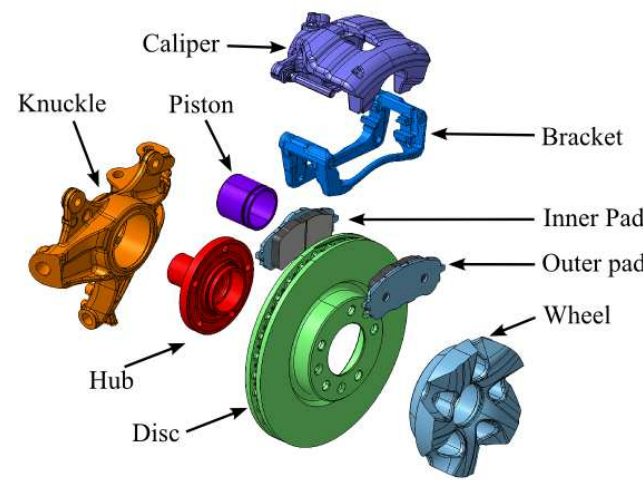

(a) Exploded view

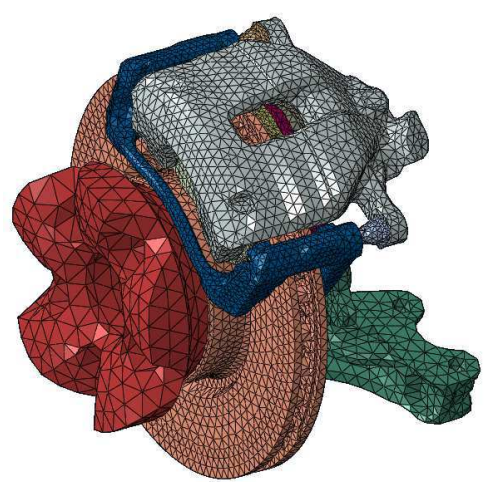

(b) Finite element model

Fig. 1. Brake system details 


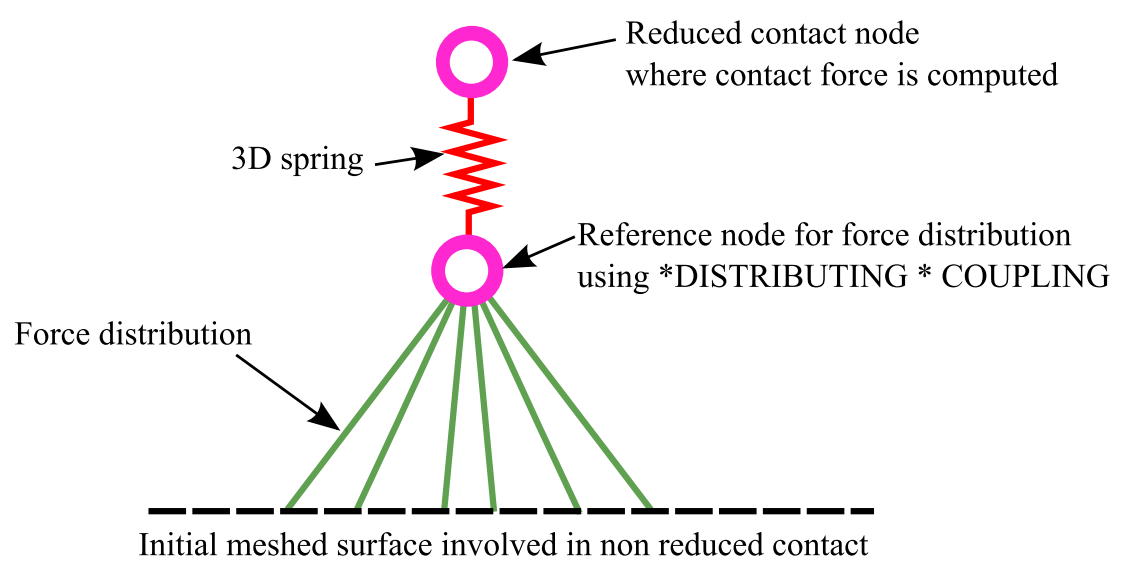

Fig. 2. The reduced contact model 


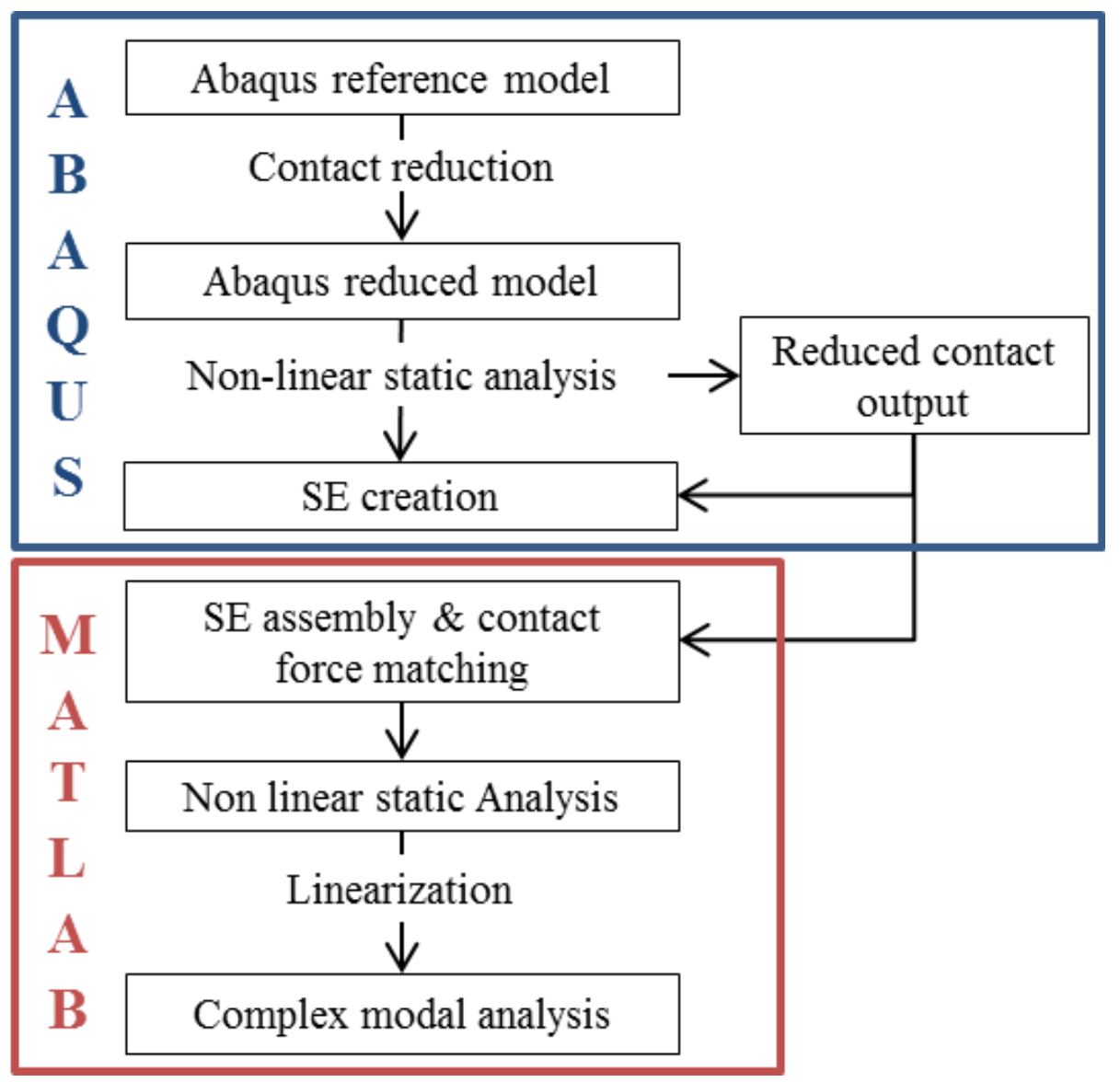

Fig. 3. Global strategy 


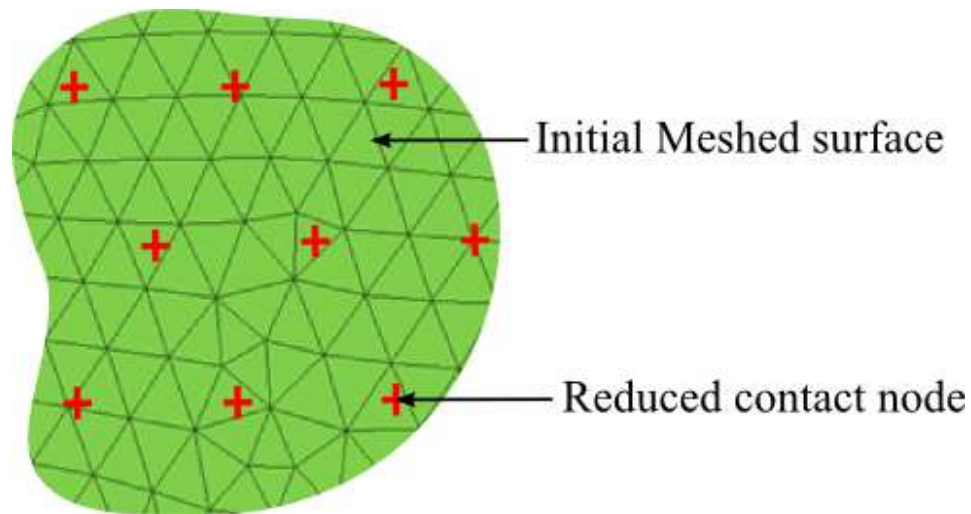

Fig. 4. Reduced contact nodes layout 


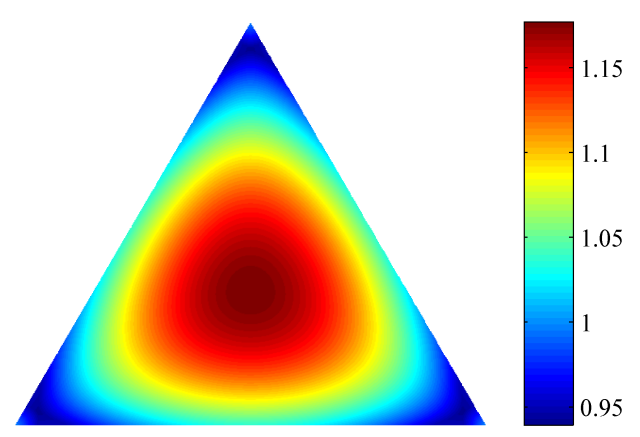

(a) $1^{\text {st }}$ degree

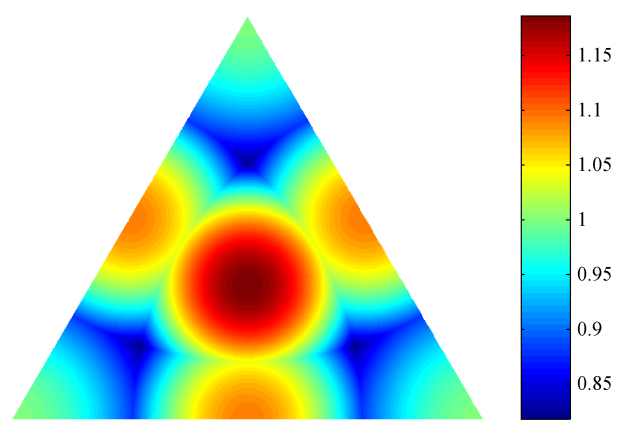

(b) $2^{\text {nd }}$ degree

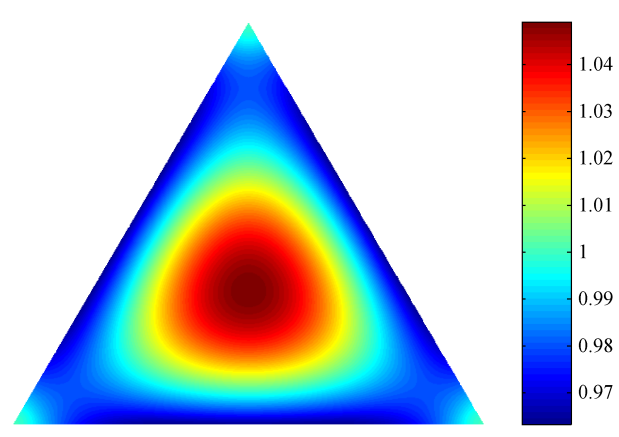

(c) $3^{\text {rd }}$ degree

Fig. 5. Weight factor distribution inside a triangle of three reduced contact nodes for several interpolation degrees. An equal force is applied on each node. 


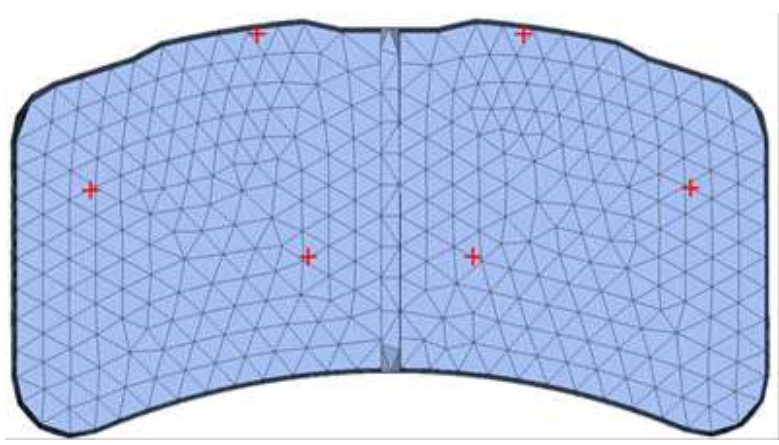

(a) 12 nodes

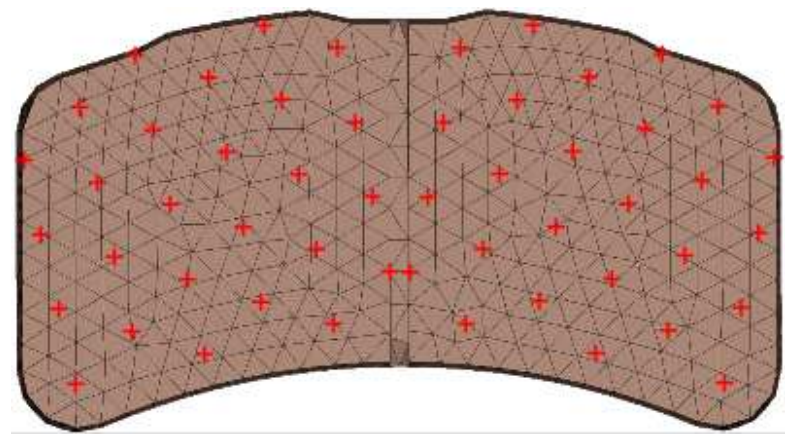

(c) 104 nodes

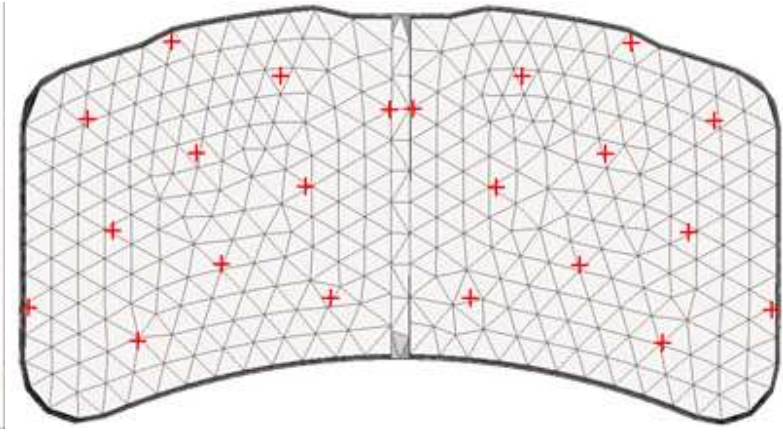

(b) 44 nodes

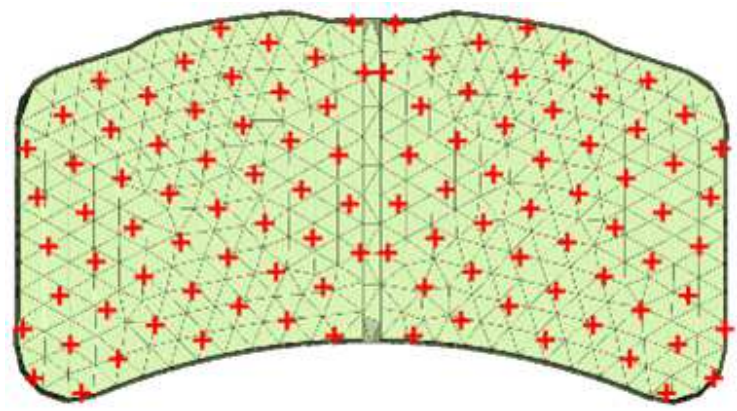

(d) 212 nodes

Fig. 6. Reduced contact nodes layout over the pad surface for several reduced model 


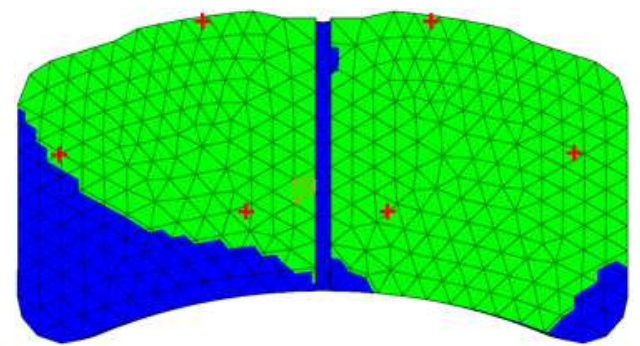

(a)

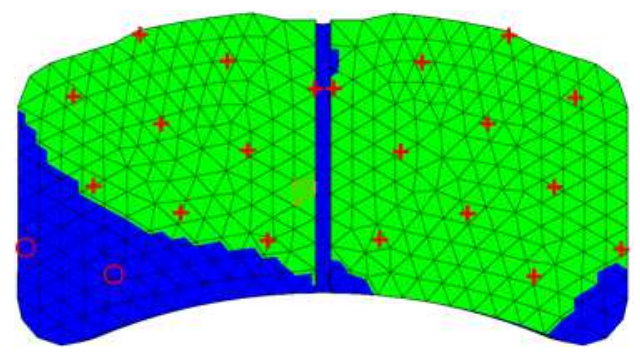

(c)

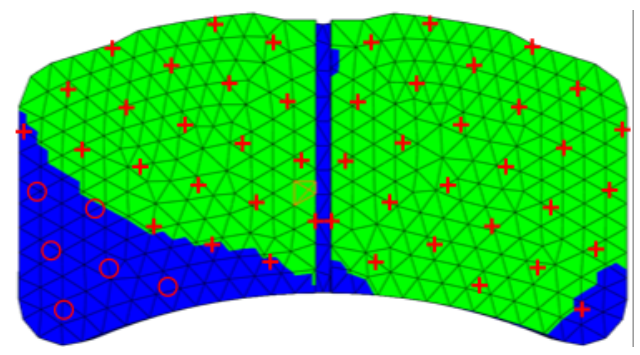

(e)

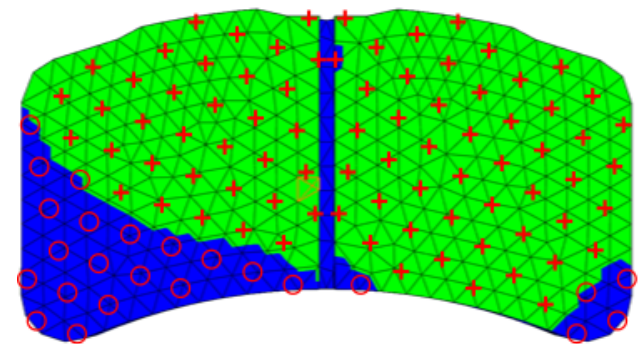

(g)

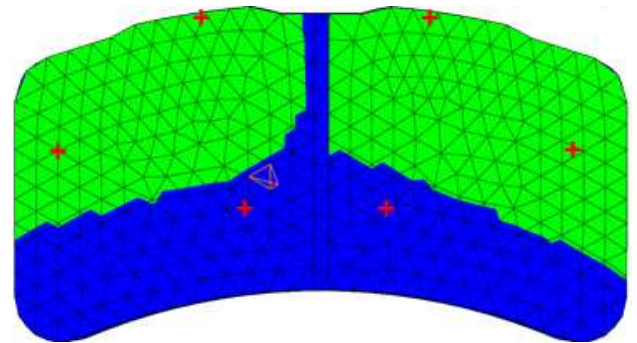

(b)

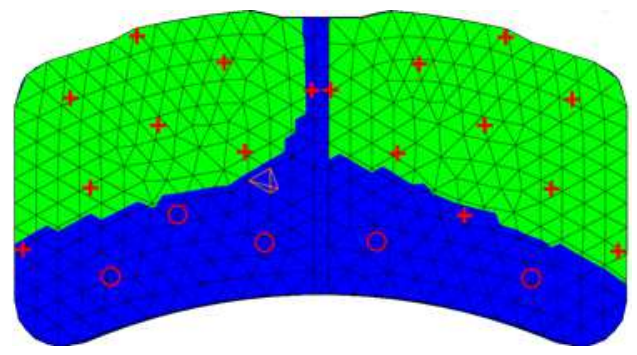

(d)

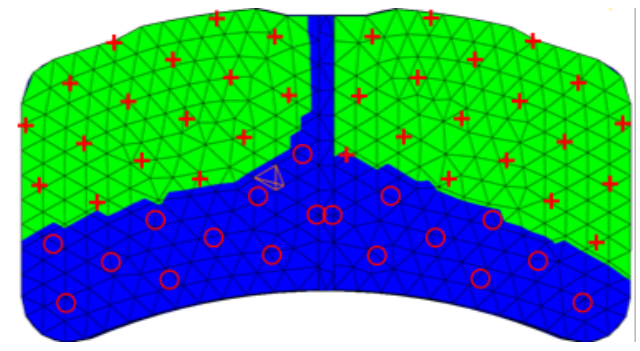

(f)

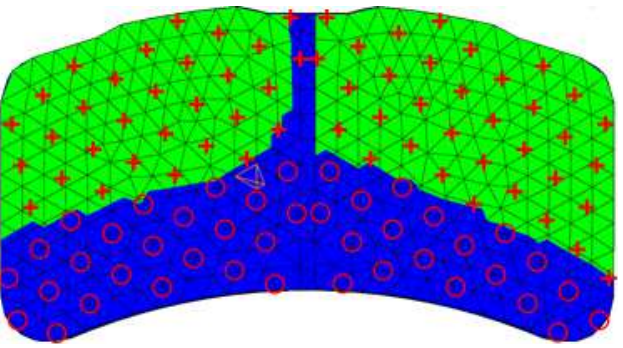

(h)

Fig. 7. Contact state comparison for each reduced model. The left column is for the inner pad and the right one is for the outer pad. (a)(b) Abaqus12 (c)(d) Abaqus44 (e)(f) Abaqus104 (g)(h) Abaqus212 


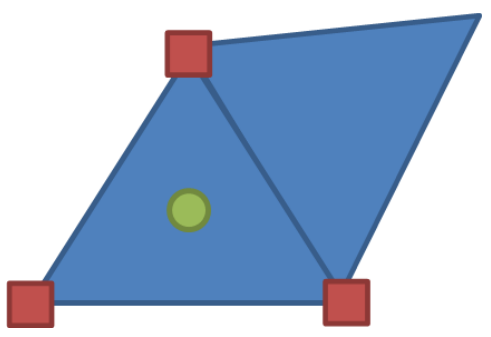

(a) Non-coincident mesh

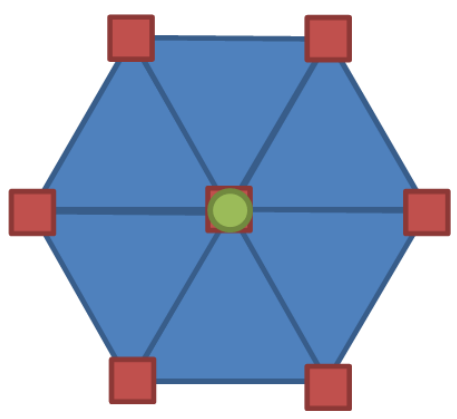

(b) Coincident mesh

Fig. 8. Contact force distribution according to the mesh coincidence. Triangles represent the master surface facets. The circle corresponds to the projection of a slave node on the master surface. Squares shows the master nodes where a contact force is computed. 


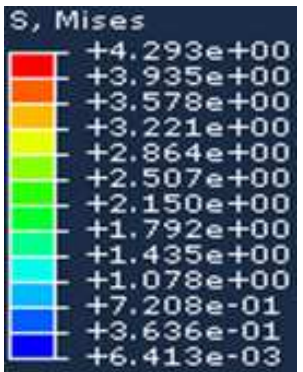

(a)

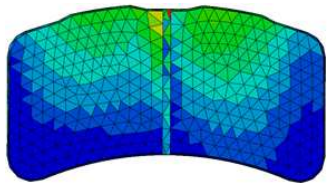

(e)

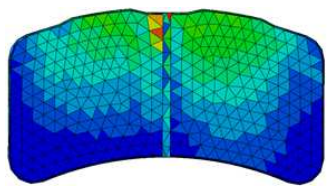

(i)

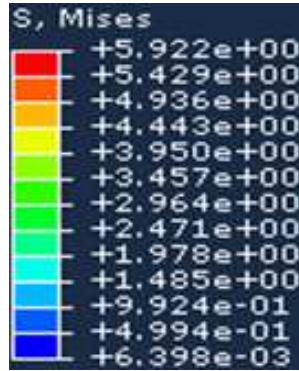

(b)

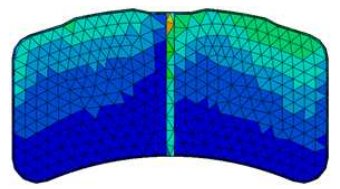

(f)

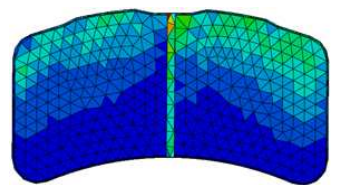

(j)

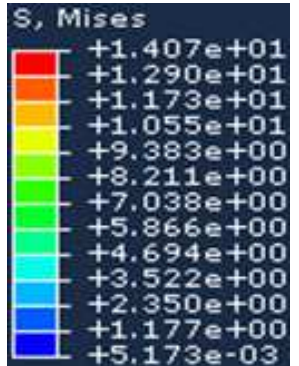

(c)

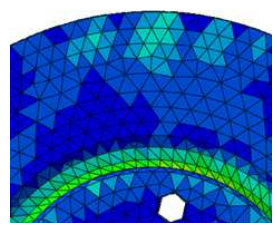

(g)

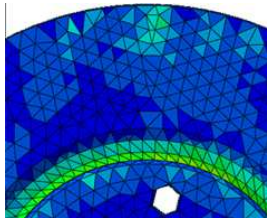

(k)

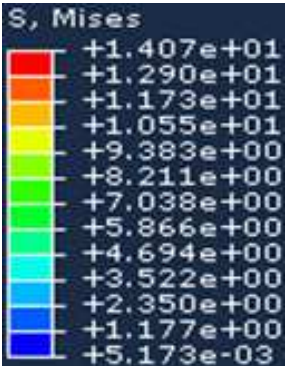

(d)

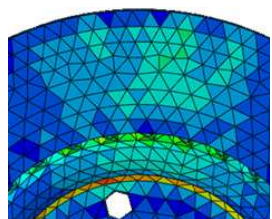

(h)

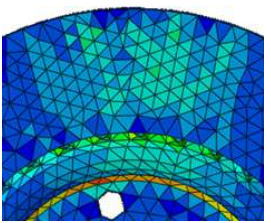

(l)

Fig. 9. Constraint field comparison between Abaqus reference model (e-h) and Abaqus212 (i-l) for disc/pad interface friction coefficient $\mu=0.5$ - (e)(i) Outer pad (f)(j) Inner pad (g)(k) Outer disc surface (h)(l) Inner disc surface. Results are given in Mpa (a-d). 


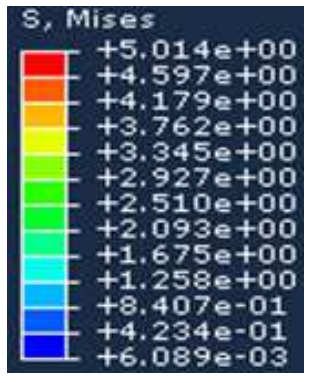

(a)

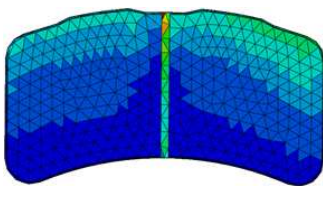

(c)

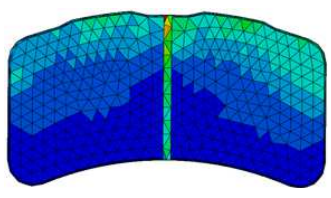

(e)

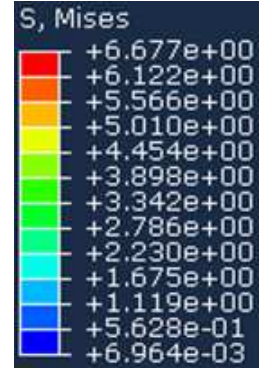

(b)

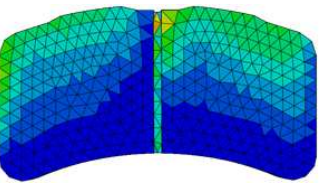

(d)

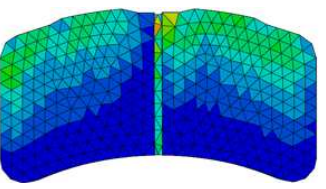

(f)

Fig. 10. Constraint field comparison at the inner pad between Abaqus reference model (c-d) and Abaqus212 (e-f) for disc/pad interface friction coefficient $\mu=0.2$ - (c-e) and $\mu=0.8$ (d-f) - results are given in MPa (a-b). 


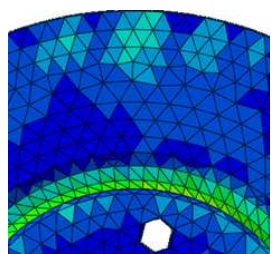

(a)

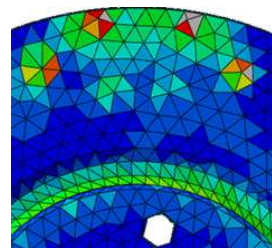

(b)

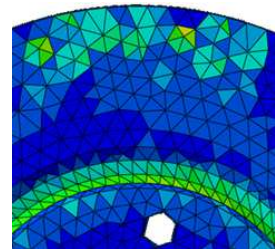

(c)

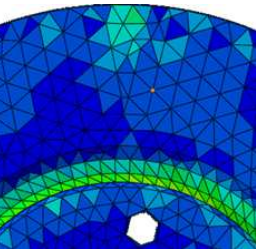

(d)

Fig. 11. Vom Mises stress comparison on outer disc surface for $\mu=0.5$ (a) Abaqus reference, (b) Abaqus12, (c) Abaqus44, (d) Abaqus104. Results are given in MPa (see Figure 9(c) for reference). 


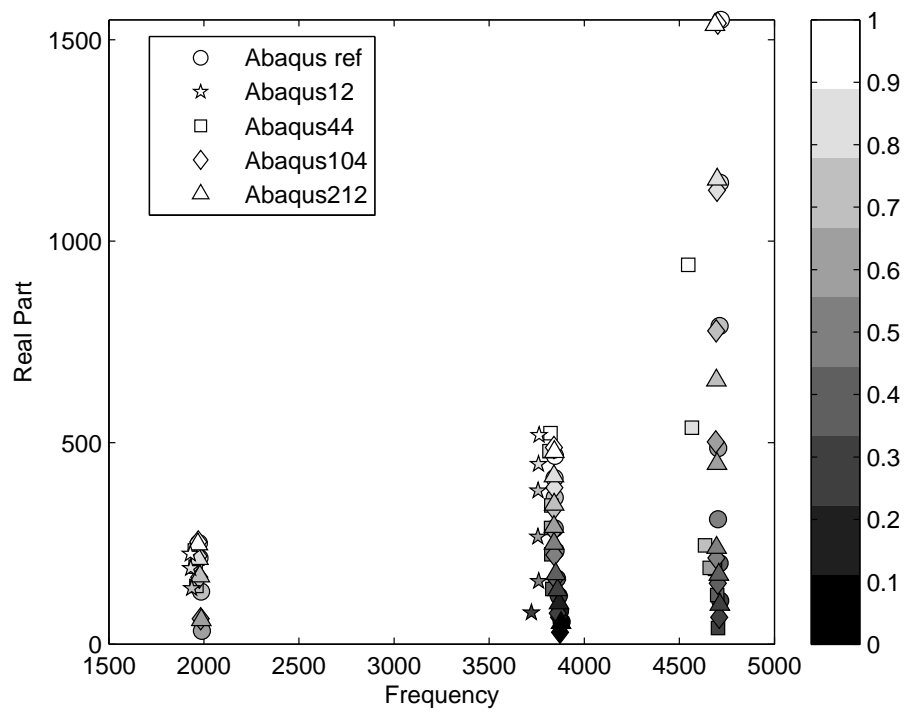

Fig. 12. Evolution of real part and frequency of the instabilities studied against the friction coefficient for Abaqus12, Abaqus44, Abaqus104 and Abaqus212 
(a)

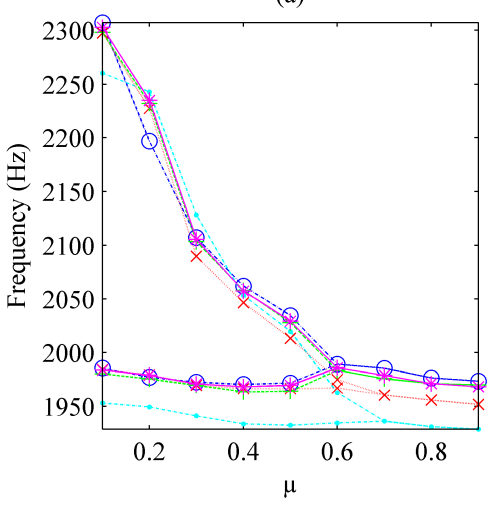

(d)

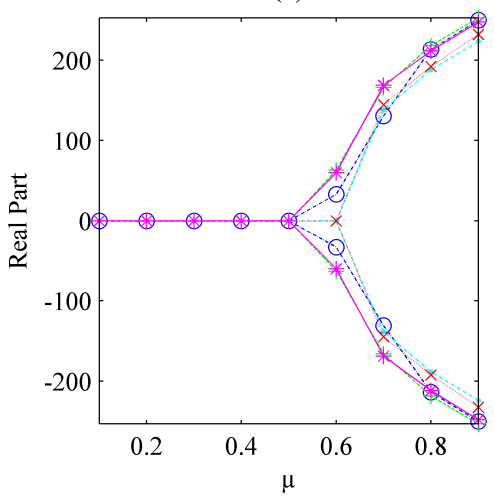

(b)

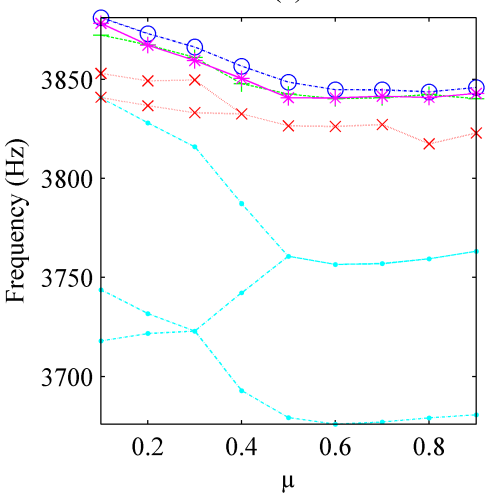

(e)

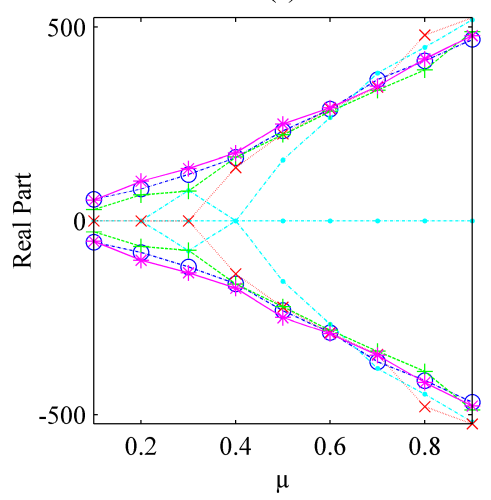

(c)

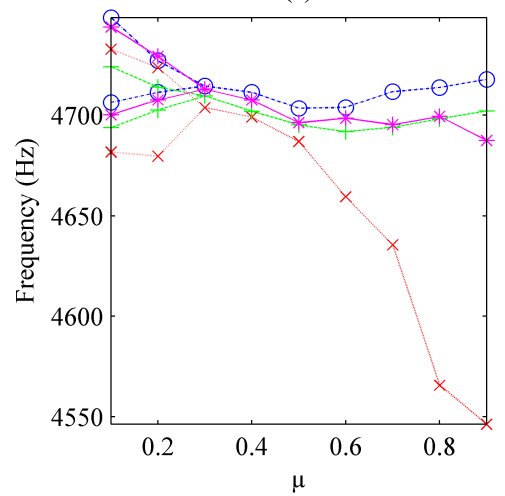

(f)

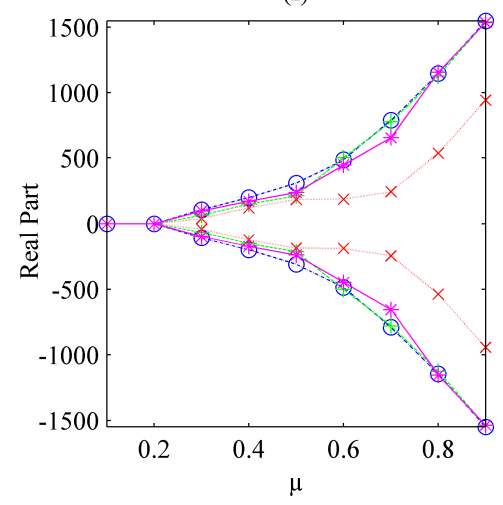

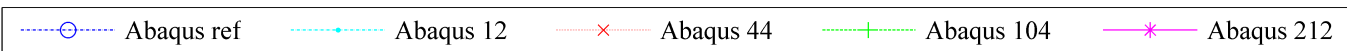

Fig. 13. Evolution of the frequencies $(a, b, c)$ and the real part (d,e,f) of the eigenvalues against the friction coefficient $\mu$ for the (a,d) $1.9 \mathrm{kHz},(\mathrm{b}, \mathrm{e}) 3.8 \mathrm{kHz}$ and $(\mathrm{c}, \mathrm{f}) 4.7 \mathrm{kHz}$ instabilities. 
(a)

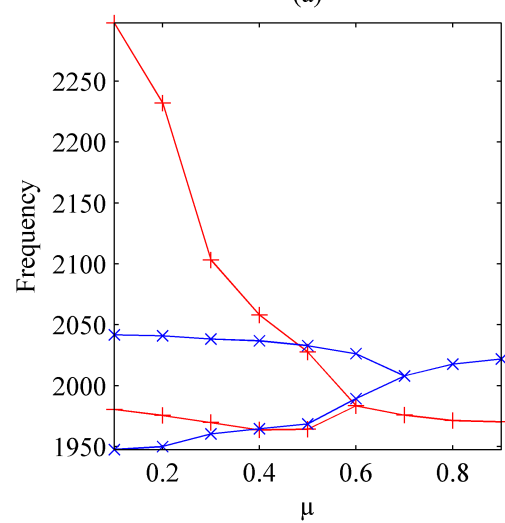

(d)

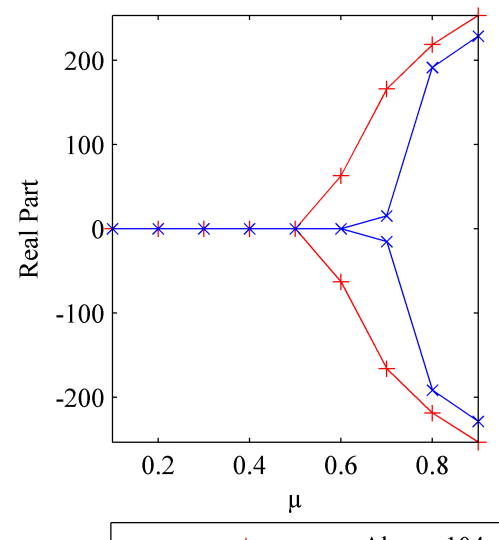

(b)

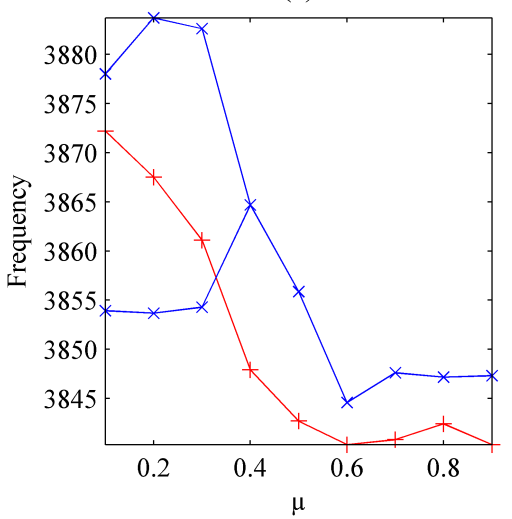

(e)

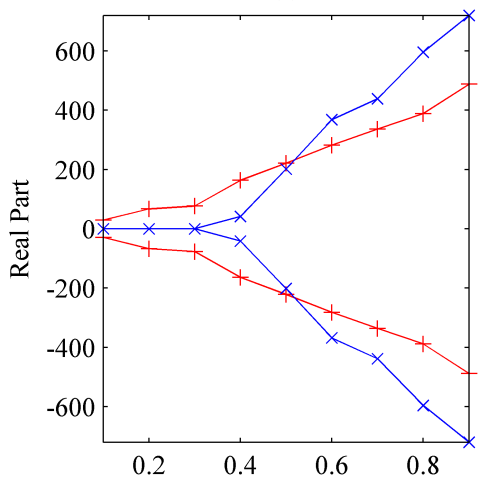

(c)

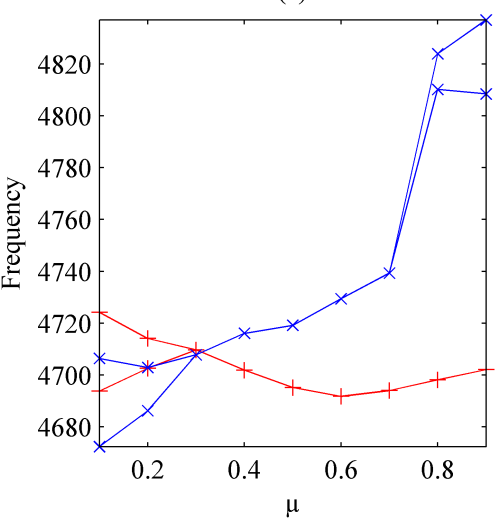

(f)

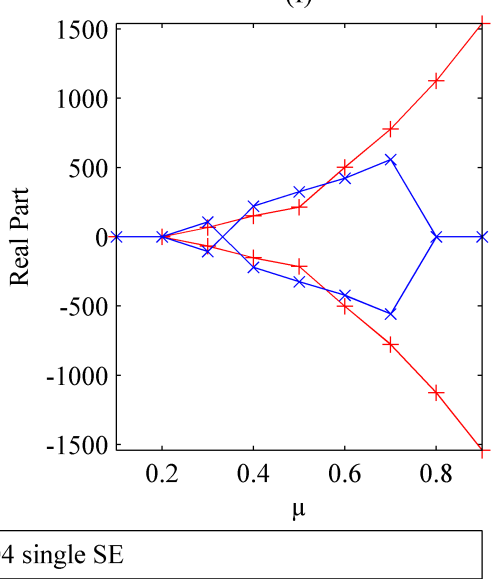

Fig. 14. Evolution of the frequencies $(\mathrm{a}, \mathrm{b}, \mathrm{c})$ and the real part $(\mathrm{d}, \mathrm{e}, \mathrm{f})$ of the eigenvalues against the friction coefficient $\mu$ for the (a,d) $1.9 \mathrm{kHz},(\mathrm{b}, \mathrm{e}) 3.8 \mathrm{kHz}$ and $(\mathrm{c}, \mathrm{f}) 4.7 \mathrm{kHz}$ instabilities. The same SuperElement generated at $\mu=0.5$ is used for the whole friction coefficient range. 


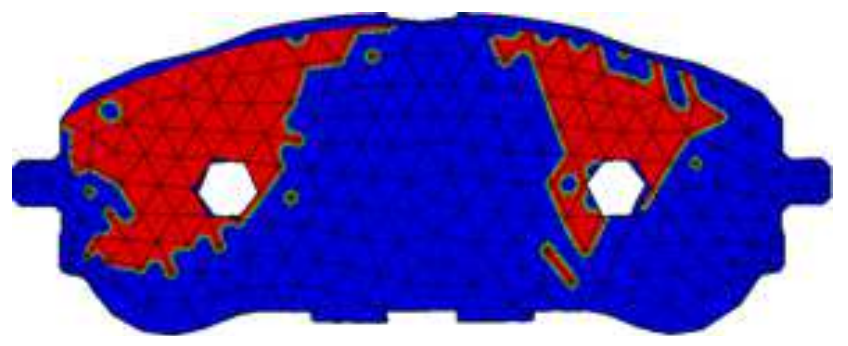

(a)

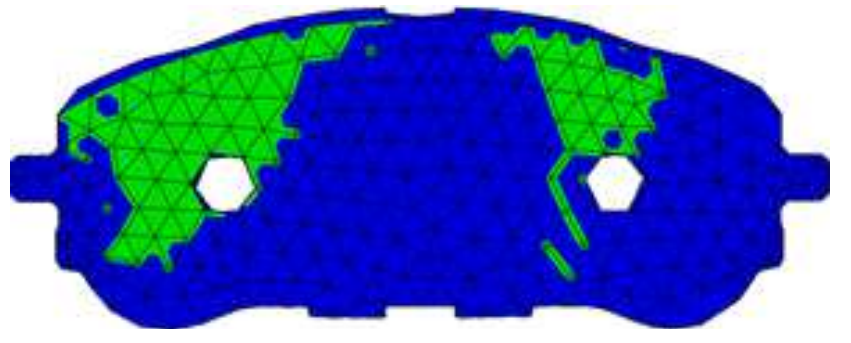

(b)

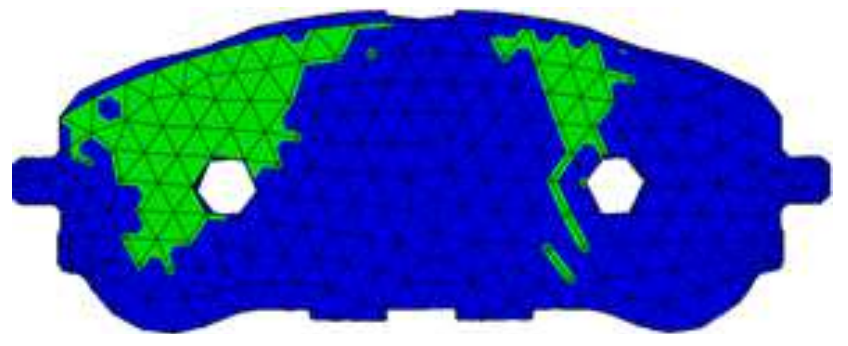

(c)

Fig. 15. Outer pad/Caliper contact status evolution on Abaqus reference model for friction coefficient value of $0.2(\mathrm{a}), 0.5(\mathrm{~b})$ and $0.8(\mathrm{c})$. Blue colour is for open contact, the others are for closed contact sticking (red) or slipping (green) 
(a)

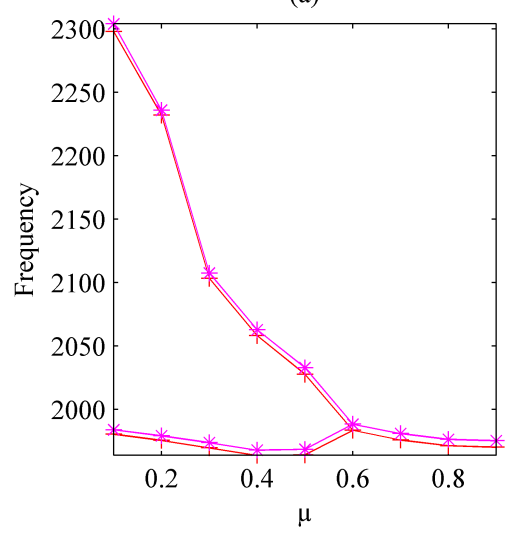

(d)

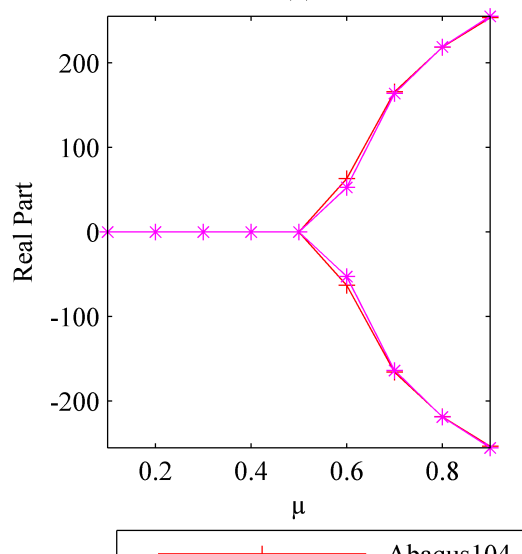

(b)

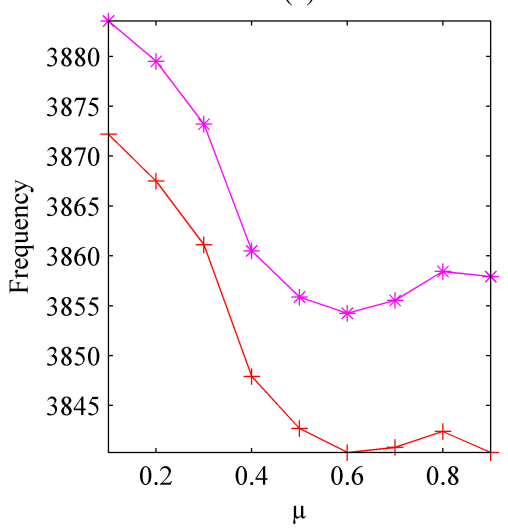

(e)

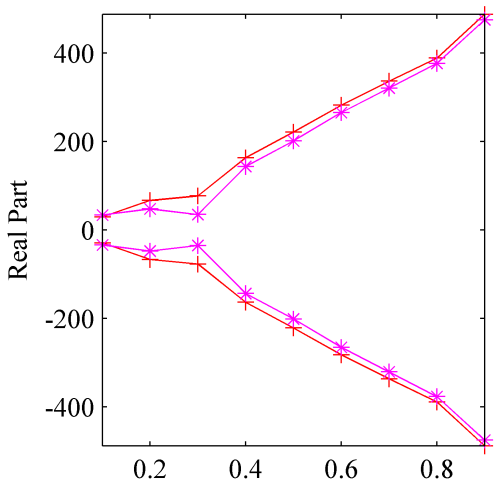

(c)

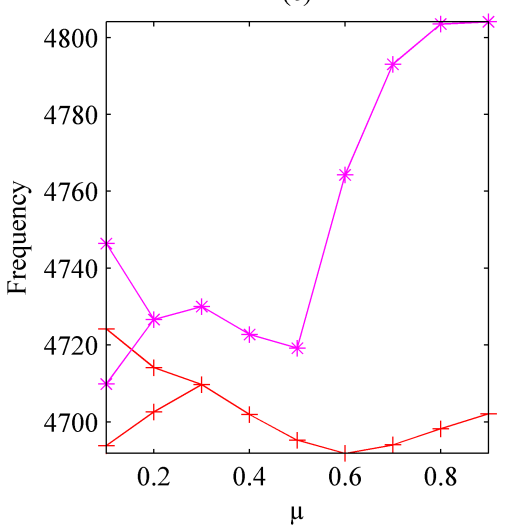

(f)

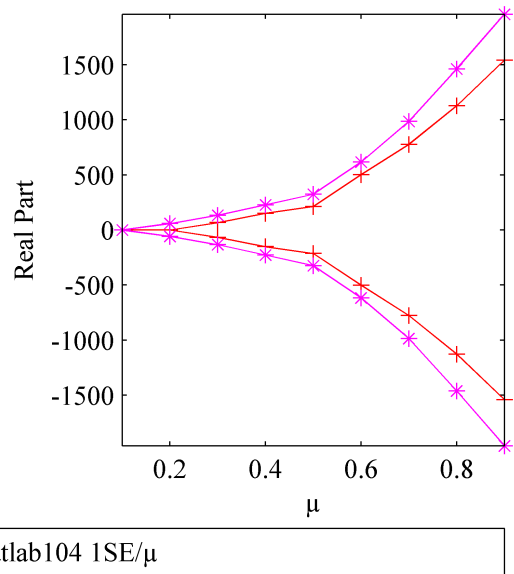

Fig. 16. Evolution of the frequencies $(a, b, c)$ and the real part $(d, e, f)$ of the eigenvalues against the friction coefficient $\mu$ for the (a,d) $1.9 \mathrm{kHz},(\mathrm{b}, \mathrm{e}) 3.8 \mathrm{kHz}$ and $(\mathrm{c}, \mathrm{f}) 4.7 \mathrm{kHz}$ instabilities. A new SuperElement is generated at each $\mu$ for complex eigenvalue analysis on $\mathrm{SE}$ assembly. 


\section{List of Tables}

$1 \quad$ Contact modelling . . . . . . . . . . . . . . . . . . . . . 40

$2 \quad$ Weight factor computation results for several interpolation degrees . . . . . . . . 41

3 Reduced models characteristics . . . . . . . . . . . . . . . . . . . . . 42

4 Mean error on frequencv. Abaqus 104/212 versus Abaqus reference . . . . . . . . 43

5 Min/Max error on frequencv. Abaqus 104/212 versus Abaqus reference . . . . . . 44

$6 \quad$ Mean error on frequencv. SE assemblv versus Abaqus 104 model . . . . . . . . . 45

7 Min/Max error on frequency. SE assembly versus Abaqus 104 model . . . . . . . 46 
Table 1

Contact modelling

\begin{tabular}{|c|c|c|}
\hline Contact & Friction coefficient & Separation \\
\hline Disc/Pad & 0.5 & Yes \\
\hline Piston/Pad & 0.15 & No \\
\hline Bracket/Pad & 0.15 & Yes \\
\hline Caliper/Pad & 0.15 & No \\
\hline
\end{tabular}


Table 2

Weight factor computation results for several interpolation degrees: Optimal Radius, mean value $(\bar{\omega})$ and standard deviation $(\sigma)$

\begin{tabular}{|c|c|c|c|}
\hline Interpolation degree & Radius $(\mathrm{mm})$ & $\bar{\omega}$ & $\sigma$ \\
\hline 1 & 0.9501 & 1 & 0.0574 \\
\hline 2 & 0.7425 & 0.9999 & 0.0835 \\
\hline 3 & 0.9597 & 1 & 0.0234 \\
\hline
\end{tabular}


Table 3

Reduced models characteristics

\begin{tabular}{|c|c|c|c|c|}
\hline & Case 1 & Case 2 & Case 3 & Case 4 \\
\hline Abaqus models & Abaqus 12 & Abaqus 44 & Abaqus 104 & Abaqus 212 \\
\hline Reduced contact nodes per pad & 6 & 22 & 52 & 106 \\
\hline Total coincident nodes on disc volume mesh & 12 & 44 & 104 & 212 \\
\hline Total references nodes for SE & 26 & 90 & 210 & 526 \\
\hline Number of nodes to node contact elements & 12 & 44 & 104 & 212 \\
\hline
\end{tabular}


Table 4

Mean error on frequency for each pair of mode on the whole friction range between Abaqus 104/212 and the Abaqus reference model

\begin{tabular}{|c|c|c|c|}
\hline Instability number & 1 & 2 & 3 \\
\hline Abaqus 104 & $0.713 \%$ & $0.289 \%$ & $0.536 \%$ \\
\hline Abaqus 212 & $0.563 \%$ & $0.247 \%$ & $0.415 \%$ \\
\hline
\end{tabular}


Table 5

Min/Max error on frequency for each pair of mode between Abaqus 104/212 and the Abaqus reference model

\begin{tabular}{|c|c|c|c|}
\hline Instability number & 1 & 2 & 3 \\
\hline Abaqus 104 & $0.332 \% / 1.688 \%$ & $0.073 \% / 0.459 \%$ & $0.208 \% / 0.779 \%$ \\
\hline Abaqus 212 & $0.152 \% / 1.821 \%$ & $0.149 \% / 0.411 \%$ & $0.064 \% / 1.293 \%$ \\
\hline
\end{tabular}


Table 6

Mean error on frequency for each pair of mode on the whole friction range between SE assembly and the Abaqus 104 model

\begin{tabular}{|c|c|c|c|}
\hline Instability number & 1 & 2 & 3 \\
\hline Matlab 104 single SE & $4.829 \%$ & $0.543 \%$ & $1.872 \%$ \\
\hline Matlab 104 SE $/ \mu$ & $0.461 \%$ & $0.713 \%$ & $2.276 \%$ \\
\hline
\end{tabular}


Table 7

Min/Max error on frequency for each pair of mode between SE assembly and the Abaqus 104 model

\begin{tabular}{|c|c|c|c|}
\hline Instability number & 1 & 2 & 3 \\
\hline Matlab 104 single SE & $0.477 \% / 12.818 \%$ & $0.223 \% / 0.873 \%$ & $0.081 \% / 5.131 \%$ \\
\hline Matlab 104 SE/ $\mu$ & $0.355 \% / 0.519 \%$ & $0.587 \% / 0.918 \%$ & $0.778 \% / 4.482 \%$ \\
\hline
\end{tabular}

